\title{
Adjustment and Coronavirus: How to Prepare for COVID-19 Pandemic- Related Adjustment Disorder Worldwide?
}

\author{
Evaldas Kazlauskas \\ Vilnius University
}

\author{
Soledad Quero \\ Jaume I University
}

\begin{abstract}
As the 2019 novel coronavirus (COVID-19) is spreading worldwide in 2020, there is a growing concern about the impact of the pandemic on mental health. Multiple stressors associated with the pandemic, such as health-related stressors, job loss, and work-related stressors, could increase the prevalence of adjustment disorders worldwide. The present article acknowledges adjustment disorder as a highly relevant mental health outcome of the pandemic that should be addressed by mental health professionals.
\end{abstract}

Keywords: COVID-19, coronavirus, adjustment disorder, adjustment, stress

As the 2019 novel coronavirus (COVID-19) is spreading worldwide in 2020 (World Health Organization [WHO], 2020), there is a growing concern about the impact of the pandemic on the mental health of individuals, communities, and societies across the globe (Adhanom Ghebreyesus, 2020; Fiorillo \& Gorwood, 2020). The COVID-19 pandemic is not only a worldwide health crisis but has a huge impact on social life, economies, political situations, and other important areas of life. The novel disease and strict public health measures trigger high levels of stress and anxiety (Li et al., 2020; Lu, Wang, Lin, \& Li, 2020; Wang et al., 2020; Zhang et al., 2020), particularly among medical staff (Lu et al., 2020; Zhang et al., 2020). However, the concepts of trauma and posttraumatic stress disorder in the current development of the pandemic cannot be applied to all (Boyraz \& Legros, 2020; Wang et al., 2020) because the majority of the population might not be threatened by illness personally but, rather, experience significant life stressors and losses, including job loss, financial insecurity, and others. We propose taking a close look at adjustment disorder as one of the most likely consequences of the pandemic on mental health. A high increase in adjustment problems in response to the stressors associated with COVID-19 could be present in the several months since the start of the pandemic and could last long after the vaccine or effective medication to cure COVID-19 becomes available. How are we going to understand and identify adjustment problems

Editor's Note. This commentary received rapid review due to the timesensitive nature of the content. It was reviewed by the Journal Editor.-KKT

This article was published Online First June 18, 2020.

(D) Evaldas Kazlauskas, Center for Psychotraumatology, Institute of Psychology, Vilnius University; (D) Soledad Quero, Department of Basic, Clinical Psychology and Psychobiology, Jaume I University.

Correspondence concerning this article should be addressed to Evaldas Kazlauskas, Center for Psychotraumatology, Institute of Psychology, Vilnius University, Ciurlionio Street 29, Vilnius 03100, Lithuania. E-mail: evaldas.kazlauskas@fsf.vu.lt related to COVID-19? And how are we going to provide psychosocial support to a global population with adjustment problems?

\section{Adjustment Disorder: Findings From Recent Research Relevant to COVID-19}

Over the last decade, a new wave of adjustment-disorder studies emerged, mostly in European countries, such as Germany, Israel, Lithuania, Spain, and Switzerland (Kazlauskas, Zelviene, Lorenz, Quero, \& Maercker, 2017), as a response to the proposal for a new definition of adjustment disorder (Maercker et al., 2013) for the 11th edition of the International Classification of Diseases (ICD11) released by the WHO (2018). In the $I C D-11$, adjustment disorder has been defined as a stress response to significant and identifiable life stressors with a clinical presentation of the following two core symptoms: (a) preoccupation with a stressor and (b) failure to adapt, which might also include sleep disorder symptoms, among others. Adjustment disorder is often viewed as a subthreshold disorder or mild disorder on the continuum of psychopathology (Zelviene \& Kazlauskas, 2018) because it is diagnosed if an individual does not meet the criteria for other mental disorders. However, it is also well known that adjustment disorder is a serious condition that is associated with higher suicide risk (Casey, Jabbar, O'Leary, \& Doherty, 2015; Killikelly et al., 2019).

Recently, new adjustment disorder measures have been developed and validated: the Adjustment Disorder New Module (ADNM; e.g., Bachem, Perkonigg, Stein, \& Maercker, 2017; Kazlauskas, Gegieckaite, Eimontas, Zelviene, \& Maercker, 2018) and the International Adjustment Disorder Questionnaire (Shevlin et al., 2020). These novel self-report measures of adjustment disorder are highly relevant because they could provide a screening of adjustment-disorder symptoms in large samples and, in the context of the COVID-19 pandemic, help identify the most vulnerable. A recent general population study in Lithuania found that workrelated stressors and health-related stressors were significant predictors of risk for adjustment disorder (Zelviene, Kazlauskas, \& Maercker, 2020). Furthermore, a study in Switzerland found that 
adjustment disorder was common after involuntary job loss (Lorenz, Perkonigg, \& Maercker, 2018).

As the coronavirus pandemic is affecting the global population, the main issue is how to ensure psychosocial support for individuals with adjustment disorder. E-health interventions emerge as a key alternative. In this line, one of the priorities for the COVID-19 pandemic very recently stated by Holmes et al. (2020) is the design, evaluation, and rollout of remotely delivered psychological interventions for vulnerable groups. Promising approaches have recently been developed that target adjustment disorder symptoms using online interventions (Lindsäter et al., 2018; Moser, Bachem, Berger, \& Maercker, 2019; Rachyla et al., 2018; Skruibis et al., 2016) or virtual reality (Quero et al., 2019). The randomized controlled trials (RCTs) testing the efficacy of Internet-delivered low-intensity interventions found moderate to large effect sizes (Eimontas, Rimsaite, Gegieckaite, Zelviene, \& Kazlauskas, 2018; Lindsäter et al., 2018; Moser et al., 2019; Quero et al., 2019). However, these studies also reported high dropout rates (Kazlauskas, Eimontas, Olff, Zelviene, \& Andersson, 2020; Moser et al., 2019). Lower dropout rates were found when therapist support was provided (Lindsäter et al., 2018). However, in one particular intervention, the Brief Adjustment Disorder Intervention (BADI), no additional beneficial effects of therapist participation were found, indicating that low-intensity self-help interventions could result in significant improvement (Eimontas, Gegieckaite, et al., 2018).

\section{Future Directions}

As researchers and clinicians from Spain in the south of Europe and Lithuania in northeastern Europe, we witness the different spread of coronavirus and the differing COVID-19 mortality rates in our countries (WHO, 2020). We see that despite differences in the confirmed cases of COVID-19, populations in Lithuania and Spain are highly affected by the pandemic, as well as in other countries worldwide. Knowledge about the impact of the pandemic on mental health is becoming available; however, effects on adjustment difficulties remain unknown. We call for action and invite researchers not to overlook adjustment disorder in relation to COVID-19. Job loss, financial difficulties, health problems, and other life stressors could be associated with a high risk of adjustment disorder during the pandemic and in the post-COVID-19 era. There are promising developments in adjustment-disorder assessment and Internet-based psychological interventions specifically targeting adjustment-disorder symptoms. It is time for mental health professionals, policymakers, and health-care systems to use that knowledge to deal with COVID-19-related adjustment disorder and apply current research findings to help populations on a large scale. Furthermore, high-quality research on e-health interventions to treat this condition is an urgent need. This research should be multidisciplinary and undertaken in international collaboration with other countries (Holmes et al., 2020).

\section{References}

Adhanom Ghebreyesus, T. (2020). Addressing mental health needs: An integral part of COVID-19 response. World Psychiatry, 19, 129-130. http://dx.doi.org/10.1002/wps.20768

Bachem, R., Perkonigg, A., Stein, D. J., \& Maercker, A. (2017). Measuring the ICD-11 adjustment disorder concept: Validity and sensitivity to change of the Adjustment Disorder-New Module Questionnaire in a clinical intervention study. International Journal of Methods in Psychiatric Research, 26, e15451. http://dx.doi.org/10.1002/mpr.1545

Boyraz, G., \& Legros, D. N. (2020). Coronavirus disease (COVID-19) and traumatic stress: Probable risk factors and correlates of posttraumatic stress disorder. Journal of Loss and Trauma. Advance online publication. http://dx.doi.org/10.1080/15325024.2020.1763556

Casey, P., Jabbar, F., O’Leary, E., \& Doherty, A. M. (2015). Suicidal behaviours in adjustment disorder and depressive episode. Journal of Affective Disorders, 174, 441-446. http://dx.doi.org/10.1016/j.jad.2014 .12 .003

Eimontas, J., Gegieckaite, G., Dovydaitiene, M., Mazulyte, E., Rimsaite, Z., Skruibis, P., . . Kazlauskas, E. (2018). The role of therapist support on effectiveness of an Internet-based modular self-help intervention for adjustment disorder: A randomized controlled trial. Anxiety, Stress \& Coping: An International Journal, 31, 146-158. http://dx.doi.org/10 $.1080 / 10615806.2017 .1385065$

Eimontas, J., Rimsaite, Z., Gegieckaite, G., Zelviene, P., \& Kazlauskas, E. (2018). Internet-based self-help intervention for ICD-11 adjustment disorder: Preliminary findings. Psychiatric Quarterly, 89, 451-460. http:// dx.doi.org/10.1007/s11126-017-9547-2

Fiorillo, A., \& Gorwood, P. (2020). The consequences of the COVID-19 pandemic on mental health and implications for clinical practice. European Psychiatry, 63, e32. http://dx.doi.org/10.1192/j.eurpsy.2020.35

Holmes, E. A., O'Connor, R. C., Perry, V. H., Tracey, I., Wessely, S., Arseneault, L., . . . Bullmore, E. (2020). Multidisciplinary research priorities for the COVID-19 pandemic: A call for action for mental health science. The Lancet Psychiatry, 7, 547-560. http://dx.doi.org/10 .1016/S2215-0366(20)30168-1

Kazlauskas, E., Eimontas, J., Olff, M., Zelviene, P., \& Andersson, G. (2020, March). Adherence predictors in Internet-delivered self-help intervention for life stressors-related adjustment disorder. Frontiers in Psychiatry, 11, 137. http://dx.doi.org/10.3389/fpsyt.2020.00137

Kazlauskas, E., Gegieckaite, G., Eimontas, J., Zelviene, P., \& Maercker, A. (2018). A brief measure of the International Classification of Diseases-11 adjustment disorder: Investigation of psychometric properties in an adult help-seeking sample. Psychopathology, 51, 10-15. http://dx.doi.org/10.1159/000484415

Kazlauskas, E., Zelviene, P., Lorenz, L., Quero, S., \& Maercker, A. (2017). A scoping review of ICD-11 adjustment disorder research. European Journal of Psychotraumatology, 8, 1421819. http://dx.doi.org/10.1080/ 20008198.2017.1421819

Killikelly, C., Lorenz, L., Bauer, S., Mahat-Shamir, M., Ben-Ezra, M., \& Maercker, A. (2019). Prolonged grief disorder: Its co-occurrence with adjustment disorder and post-traumatic stress disorder in a bereaved Israeli general-population sample. Journal of Affective Disorders, 249, 307-314. http://dx.doi.org/10.1016/j.jad.2019.02.014

Li, Y., Wang, Y., Jiang, J., Valdimarsdóttir, U. A., Fall, K., Fang, F., . . . Zhang, W. (2020). Psychological distress among health professional students during the COVID-19 outbreak. Psychological Medicine. Advance online publication. http://dx.doi.org/10.1017/S00332917 20001555

Lindsäter, E., Axelsson, E., Salomonsson, S., Santoft, F., Ejeby, K., Ljótsson, B., . . . Hedman-Lagerlöf, E. (2018). Internet-based cognitive behavioral therapy for chronic stress: A randomized controlled trial. Psychotherapy and Psychosomatics, 87, 296-305. http://dx.doi.org/10 $.1159 / 000490742$

Lorenz, L., Perkonigg, A., \& Maercker, A. (2018). The course of adjustment disorder following involuntary job loss and its predictors of latent change. Clinical Psychological Science, 6, 647-657. http://dx.doi.org/ 10.1177/2167702618766290

Lu, W., Wang, H., Lin, Y., \& Li, L. (2020). Psychological status of medical workforce during the COVID-19 pandemic: A cross-sectional study. 
Psychiatry Research, 288, 112936. http://dx.doi.org/10.1016/j.psychres .2020 .112936

Maercker, A., Brewin, C. R., Bryant, R. A., Cloitre, M., van Ommeren, M., Jones, L. M., . . . Reed, G. M. (2013). Diagnosis and classification of disorders specifically associated with stress: Proposals for ICD-11. World Psychiatry, 12, 198-206. http://dx.doi.org/10.1002/wps.20057

Moser, C., Bachem, R., Berger, T., \& Maercker, A. (2019). ZIEL: Internetbased self-help for adjustment problems: Results of a randomized controlled trial. Journal of Clinical Medicine, 8, 1655. http://dx.doi.org/10 $.3390 / \mathrm{jcm} 8101655$

Quero, S., Molés, M., Campos, D., Andreu-Mateu, S., Baños, R. M., \& Botella, C. (2019). An adaptive virtual reality system for the treatment of adjustment disorder and complicated grief: 1-year follow-up efficacy data. Clinical Psychology \& Psychotherapy, 26, 204-217. http://dx.doi .org/10.1002/cpp. 2342

Rachyla, I., Pérez-Ara, M., Molés, M., Campos, D., Mira, A., Botella, C., \& Quero, S. (2018). An Internet-based intervention for adjustment disorder (TAO): Study protocol for a randomized controlled trial. $B M C$ Psychiatry, 18, 161. http://dx.doi.org/10.1186/s12888-018-1751-6

Shevlin, M., Hyland, P., Ben-Ezra, M., Karatzias, T., Cloitre, M., Vallières, F., . . Maercker, A. (2020). Measuring ICD-11 adjustment disorder: The development and initial validation of the International Adjustment Disorder Questionnaire. Acta Psychiatrica Scandinavica, 141, 265-274. http://dx.doi.org/10.1111/acps.13126

Skruibis, P., Eimontas, J., Dovydaitiene, M., Mazulyte, E., Zelviene, P., \& Kazlauskas, E. (2016). Internet-based modular program BADI for adjustment disorder: Protocol of a randomized controlled trial. BMC Psychiatry, 16, 264. http://dx.doi.org/10.1186/s12888-016-0980-9
Wang, C., Pan, R., Wan, X., Tan, Y., Xu, L., McIntyre, R. S., . . Ho, C. (2020). A longitudinal study on the mental health of general population during the COVID-19 epidemic in China. Brain, Behavior, and Immunity. Advance online publication. http://dx.doi.org/10.1016/j.bbi.2020 .04 .028

World Health Organization. (2018). International classification of diseases for mortality and morbidity statistics (11th revision). Retrieved from https://icd.who.int/browse11/1-m/en

World Health Organization. (2020). Coronavirus (COVID-19) dashboard. https://covid19.who.int/

Zelviene, P., \& Kazlauskas, E. (2018). Adjustment disorder: Current perspectives. Neuropsychiatric Disease and Treatment, 14, 375-381. http:// dx.doi.org/10.2147/NDT.S121072

Zelviene, P., Kazlauskas, E., \& Maercker, A. (2020). Risk factors of ICD-11 adjustment disorder in the Lithuanian general population exposed to life stressors. European Journal of Psychotraumatology, 11, 1708617. http://dx.doi.org/10.1080/20008198.2019.1708617

Zhang, W.-R., Wang, K., Yin, L., Zhao, W.-F., Xue, Q., Peng, M., . . . Wang, H.-X. (2020). Mental health and psychosocial problems of medical health workers during the COVID-19 epidemic in china. Psychotherapy and Psychosomatics, 1, 1-9. http://dx.doi.org/10.1159/ 000507639

Received April 26, 2020

Revision received May 24, 2020

Accepted May 27, 2020 